\title{
GUÍA COMUNICATIVA PARA INTERLOCUTORES-CLAVE EN EL MARCO DE LA CONVERSACIÓN AFÁSICA
}

\author{
Beatriz Gallardo Paúls \\ Universitat de València - Estudi General \\ Beatriz.Gallardo@uv.es
}

\begin{abstract}
Resumen
Con este trabajo presentamos una Guía comunicativa diseñada para los interlocutores-clave (Whitworth, Perkins y Lesser 1997) que participan en conversaciones donde se incluye algún hablante con afasia. La necesidad de este tipo de guías surgió durante la elaboración del corpus PerLA ("PERcepción, Lenguaje y Afasia"), iniciado en 2000 en el área de Lingüistica General de la UVEG. Presentamos algunos trabajos anteriores que abordan el tema de los interlocutores, como el Protocolo Pragmático diseñado por Carol Prutting y Diane Kirchner, el Entrenamiento conversacional de Audrey Holland, y la Terapia de Conversación Asistida de Aura Kagan. Los datos confurman que en estas situaciones ambos tipos de hablantes asumen una mayor responsabilidad en la gestión dialógica para el hablante sin afasia; a fin de ilustrar esta mayor responsabilidad y el papel esencial del interlocutor-clave en la negociación de los significados, nuestro trabajo focaliza dos conductas conversacionales centrales: los solapamientos y el sistema de toma de turno. Por último, presentamos una iniciativa que sirve de marco general para la práctica de estas habilidades conversacionales: los Cursos de Habilidades Comunicativas para Interlocutores-Clave realizados en hospitales y centros de rehabilitación.

PALABRAS CLAVE: Afasia. Eficacia conversacional. Lingüistica de corpus. Neurolingüistica. Pragmática.
\end{abstract}

\begin{abstract}
The aim of this paper is to present a Communicative Guide designed for "key conversational partners" (Whitworth, Perkins y Lesser 1997) in the context of "aphasic conversation". The need for this kind of work became evident when we started collecting data for the conversational corpus of aphasia named "PerLA" ("PERception, Language and Aphasia"), in 2000. This paper draws on prior research that deals with conversational partners, such as the Pragmatic Protocol of Carol Prutting and Diane Kirchner, the Conversational Training of Audrey Holland, and, specially, the Supported Conversation Therapy of Aura Kagan. The data supported the idea that, in contexts of aphasia, both aphasic and non-aphasic participants assume that the management of dialogue is, basically, the responsibility of the non-aphasic partner. To illustrate this responsibility and the essential role of the key conversational partner in the negotiation of meaning in conversation, the paper focuses on two emblematic conversational behaviours: overlaps and the turn-taking system. Finally, the paper highlights the usefulness of a method designed to practice these skills, namely, the Courses of Communicative Abilities for Key Conversational Partners at hospitals.
\end{abstract}

KEY WORDS: Aphasia. Conversational eficiency. Corpus linguistics. Neurolinguistics. Pragmatics.

\section{Presentación}

Los datos a partir de los que hemos preparado este trabajo proceden del corpus PerLA (Percepción, Lenguaje y Afasia), que elaboramos en el área de Lingüística General de la UVEG desde el año 2000, gracias a los proyectos de investigación "Estudio de variables morfológicas y sintáctico-semánticas en la evaluación de afasias" (BFF2001-3234-c02-02), 
"Estudio de las variables pragmáticas en la evaluación de afasias", y "Elaboración y análisis pragmático de un corpus de lenguaje afásico" (BFF2002-00349, con la ayuda complementaria de la GV, Grupos2004/14), y a los convenios de colaboración firmados con el Servicio de Neurología del Hospital Clínico de Valencia (neurólogos José Miguel Láinez y Dolores Alonso) y el Servicio de Daño Cerebral del Hospital Valencia al Mar (logopeda Raúl Villodre, doctores Javier Chirivella y Enrique Noé). Dejamos constancia de nuestra gratitud hacia estos profesionales $\mathrm{y}$, sobre todo, hacia los hablantes con afasia que acceden a participar en nuestra investigación.

En la actualidad el corpus consta de 33 grabaciones digitales de una duración aproximada de 55 minutos; existen tres tipos básicos de grabaciones:

- Una vez que el neurólogo ha informado al paciente de nuestro proyecto y este ha accedido a colaborar, realizamos grabaciones mantenidas en su domicilio; la cita se fija mediante una o varias llamadas telefónicas previas al interlocutor-clave. En algún caso, una vez acordada la cita, el paciente no ha querido participar y nos hemos ido de su casa sin más. Interviene el hablante afásico, el o los interlocutores clave, y el o los entrevistadores (nunca más de dos).

- En otros casos, la conversación se desarrolla durante la sesión de rehabilitación en el despacho del logopeda del hospital Valencia al Mar de Hospitales NISA (logopeda Raúl Villodre); la situación se plantea en todo caso como "una charla informal", no hay conductas planificadas salvo, en algunas ocasiones, la previsión de posibles temas. A veces hemos grabado la conversación entre dos hablantes con afasia.

- Un tercer tipo de grabaciones está formado por conversaciones en las que no participa el entrevistador ni el logopeda; el hablante afásico y su interlocutor clave mantienen una charla en una sala del Hospital Aguas Vivas (Hospitales NISA), habiendo recibido por parte de la logopeda (Lourdes Brines) la única instrucción de mantener una conversación informal. La realización de este tipo de grabaciones tiene por finalidad la posterior aplicación del Protocolo Pragmático (Prutting y Kirchner, 1983).

La afasia se define como una patología del lenguaje ocasionada por alguna lesión en el hemisferio izquierdo del cerebro; las causas básicas pueden ser un accidente cerebro-vascular (ictus o infarto cerebral, derrame, trombosis), un tumor o un traumatismo craneoencefálico.

\section{El tipo de interacción}

Las grabaciones que hemos descrito dan pie a un tipo de interacción que presenta dos diferencias básicas respecto a los estudios habituales de pragmática interaccional:

1. La naturaleza del encuentro y su efecto en la conducta que muestran los hablantes. Los acontecimientos comunicativos que hemos grabado tienen una naturaleza conversacional, pero con dos tipos de interlocutores claramente diferentes: el informante afásico y su familiar o familiares, y el o los entrevistadores. Y no existe entre estos dos grupos más historia conversacional (Golopentjia 1988) que las conversaciones telefónicas mantenidas a veces entre un entrevistador y un familiar para establecer la cita; en algunos casos, ni siquiera existe esta charla previa, pues un logopeda ha establecido la cita por separado con la entrevistadora y con los informantes. Este desconocimiento previo afecta a la naturaleza de los temas tratados y, obviamente, al conocimiento compartido, imponiendo cierto grado de 
formalidad. Además, dado el motivo que origina la grabación, parece imponerse como tema inevitable el tratamiento de la afasia, las causas que la han provocado y, por extensión, las diferencias entre el antes y el después de la lesión en la vida del informante; esto significa la creación de un clima que hemos llamado de delicadeza emocional, que también debe ser tenido en cuenta en la medida en que no es prototípico. Por otro lado, la paradoja del observador (Labov 1972) impone también un ligero condicionamiento que hace a los participantes más pendientes de su actividad verbal (propiciando temas metacomunicativos). En la mayor parte de los casos, se intenta que los entrevistadores participen lo mínimo posible en la conversación, pero esto no siempre es fácil; cuando hemos grabado más de una vez a un hablante, esta situación de desconocimiento se suaviza, obviamente, y además ya no resulta obligatorio contar los detalles dolorosos que han llevado a la afasia; paralelamente, puesto que la situación pierde la formalidad del primer encuentro, el entrevistador se ve más obligado a participar en el diálogo y también es habitual que el interlocutor-clave intervenga más (a veces llegando a monopolizar el turno). En la medida en que nuestros datos proceden de estas grabaciones, con la presencia de la cámara y de un participante extraño, adquieren cierta artificialidad que los aproxima al discurso público, algo que puede afectar indirectamente a cómo los participantes se comportan. Para Sennet 'La conducta 'pública' es, en primera instancia, una cuestión de acción a distancia del yo, de su historia inmediata, de sus circunstancias y necesidades". (1976: 113). Este distanciamiento del yo propio del discurso público es llamado por Barthes (1973) "borrado de sujeto"; cuando un emisor accede a la voz pública ha de asumir un lenguaje unitario, desprovisto de las marcas de sujeto que utiliza en sus comunicaciones no públicas (también Baudrillard 1987 utilizaba este concepto para referirse a la irrupción mediática de los temas privados).

2. La competencia de los sujetos. Los participantes no están en igualdad de condiciones conversacionales; la conversación cotidiana puede presentar asimetrías debidas a las relaciones de poder, por ejemplo, o a las situaciones sociales, pero en este caso la asimetría afecta a la propia competencia comunicativa del interlocutor que, precisamente, es el protagonista de la grabación. Junto a las limitaciones verbales son habituales, además, limitaciones motoras como hemiplejías del lado izquierdo u otras que afectan a la comunicación no verbal. En esta situación la competencia comunicativa del sujeto queda ENMASCARADA por la afasia (Kagan 1998). O dicho en otros términos: este hablante pasa a parecer un INCOMPETENTE (Ferguson 1996). Habida cuenta, además, de lo poco conocida que es la afasia por la opinión pública, esta incompetencia tiene todas las posibilidades de ser interpretada como una deficiencia mental, cognitiva. Se suma además a esta situación lo que Kathryn Garrett (2003) describe como INESTABILIDAD e INTERMITENCIA de esta competencia comunicativa, de tal manera que el interlocutor no puede predecir cuándo cierta conducta verbal puede causar o no un problema comunicativo.

Así pues, el juego que vamos a describir presenta diferencias fundamentales respecto a otros juegos conversacionales, pues se ven alteradas las condiciones de los hablantes y las reglas de la interacción. La comparación de la conversación con un juego, como sabemos, es frecuente en la teoría lingüística; decimos normalmente que la conversación funciona como un juego donde los participantes comparten un conocimiento de intenciones y objetivos, y eligen movimientos o jugadas estratégicas según unas decisiones racionales. Pero en las grabaciones que manejamos esas reglas se han perdido, y los participantes ignoran cómo pueden establecer nuevas reglas; la situación nos ha recordado a veces los juegos infantiles en 
que se han perdido las fichas y, sobre la marcha, los jugadores inventan reglas que permitan seguir jugando sin las fichas perdidas... porque de lo que se trata es de seguir jugando.

Así pues, ¿podemos hablar de "reglas del juego" en la conversación afásica o es todo tan caótico como a veces parece?, ¿las "reglas perdidas" son todas o sólo algunas?, ¿cuáles se mantienen y cuáles han de ser redefinidas?, ¿puede el lingüista ayudar a los interlocutores afásicos y no afásicos a explicitar tales reglas?

Para dar respuesta las preguntas que acabamos de plantear, nuestro centro de interés no será sólo la actividad subjetiva del hablante afásico, sino sobre todo la actividad del que en pragmática clínica llamamos interlocutor-clave (key conversational partner, Whitworth, Perkins y Lesser 1997), es decir, la persona que a partir del accidente se ha convertido en cuidadora y casi mediadora del hablante con el mundo. Esta figura es esencial para la investigación en dos aspectos:

- por un lado, como informante de las deficiencias ${ }^{l}$ que sufre el afásico y

- por otro lado, como agente facilitador de la eficacia comunicativa mediante el desarrollo de estrategias compensatorias. Efectivamente, podemos comprobar en las grabaciones que, mientras que por un lado existen conductas en el interlocutor-clave que pueden resultar contraproducentes para el hablante afásico, existen otras que, por el contrario, resultan altamente facilitadoras.

Así pues, a partir de la conducta conversacional que construyen ambos interlocutores, pretendemos extraer conclusiones en dos direcciones:

- las estrategias compensatorias que se desarrollan en cada caso pueden analizarse y ver su posible aplicación sistemática para otros interlocutores-clave,

- las conductas amenazantes son aisladas y objetivadas a fin de lograr la concienciación por parte de los implicados y, en consecuencia, su eliminación en la medida de lo posible (en pragmática interaccional hablamos de "conductas amenazantes" en el sentido de "amenaza para la imagen", en la línea clásica de Brown-Levinson 1978 y sus teorías sobre la cortesía, según las cuales todo acto de habla es un potencial acto de amenaza de la imagen de los hablantes).

\section{La "conversación afásica"}

Cuando nos planteamos la elaboración del corpus de lenguaje afásico, la idea fundamental que nos guiaba era la necesidad de obtener datos con "validez ecológica" (Ahlsén 1995; Joanette y Ansaldo 1999; Perkins, Withworth y Lesser 1998), es decir, acordes con las exigencias de la pragmática. Teníamos claro que necesitábamos superar la artificialidad de las situaciones asimétricas propias de la consulta, y que para ello era fundamental poder acceder al entorno habitual del hablante, y lograr datos de ocurrencia real. En estos planteamientos iniciales, sin embargo, nuestro foco de interés era en todo momento la conducta de los hablante afásicos; queríamos comprobar, como hemos señalado en otras ocasiones,

1 La OMS, en su CIDDM (Clasificación Internacional de Deficiencias, Discapacidades y Minusvalias), distingue entre tres categorias:

-- déficit o deficiencia (impairment), que se da en la estructura corporal o en la función de un órgano o sistema

- discapacidad (disability), que atañe al rendimiento funcional y a la actividad del individuo como consecuencia del déficit

- minusvalía (handicap), que tiene que ver con las limitaciones sociales y relacionales derivadas. 
hasta qué punto la pragmática estaba preservada y de qué manera podríamos rentabilizarla luego en el diseño de protocolos de rehabilitación. Nuestra idea de partida era (no la hemos perdido) poder utilizar las categorías pragmáticas básicas (presumiblemente localizadas en el hemisferio derecho) para la recuperación de las categorías gramaticales alteradas por la lesión en el hemisferio izquierdo.

A partir del estado actual de análisis de la Base de datos PerLA ${ }^{2}$, observamos que esta premisa es bastante asumible para los hablantes con afasia no fluente, pero que necesita muchas matizaciones en los hablantes con afasia fluente o sensitiva, debido a la implicación del componente semántico. Así, comprobamos que las categorías enunciativas fundamentales (actos de habla e implicaturas) se hallan disponibles en el hablante con afasia, o que, por ejemplo, los hablantes con afasia de Broca (limitaciones de producción) tienden a una sobreexplotación de las implicaturas, confiando en que la capacidad inferencial del interlocutor deducirá gran parte de lo que intenta transmitir. Para el hablante afásico fluente la observación de las máximas conversacionales o la gestión temática plantean, sin embargo, mayores problemas.

Pero la observación de los datos que hemos ido grabando, y nuestra propia participación en las grabaciones, provocó la aparición de un nuevo foco de interés: el interlocutor-clave y su conducta. Por eso hemos optado por hablar en todo momento de "conversación afásica" (Gallardo 2003); para nosotros la patología no es, tan sólo, un rasgo del individuo afectado, sino un elemento que, al trasladarse a la actividad conversacional, pasa a formar parte del acontecimiento comunicativo en su globalidad. Frente a los estudios de laboratorio más tradicionales, que focalizan "la conducta del individuo X", nosotros estamos interesados en cómo la patología afecta a todo el engranaje conversacional y comunicativo, en el sujeto y en sus interlocutores. El desarrollo de esta nueva perspectiva es lo que nos ha llevado, en última instancia, al diseño de una "Guía comunicativa" dirigida a los interlocutores habituales del hablante afásico.

\section{Antecedentes en la inclusión de los interlocutores-clave}

Hemos dicho que tomamos el concepto de "interlocutor clave" del CAPPA de Perkins, Withworth y Lesser (1997), pero la consideración de los interlocutores habituales de los hablantes con patología había aparecido anteriormente tanto en el ámbito de la evaluación como en la terapia. En los casos que mencionamos a continuación existe siempre una situación de TRIANGULACIÓN entre el hablante con patología, el logopeda/investigador y un tercer hablante:

\footnotetext{
2 Una vez damos por definitiva una transcripción (proceso harto costoso en tiempo y esfuerzo, en el que seguidmos unas convenciones de tipo etnometodológico), analizamos una serie de parámetros en una Base Access, cuyos campos incluyen datos como los siguientes: identificación, edad, sexo, nivel sociocultural estimativo, tipo de lesión cerebral, hospital de procedencia, minutos, número total de turnos, número de turnos de cada interlocutor, número total de palabras, número de palabras de cada interlocutor, tareas de edición (a partir de Crockford y Lesser 1994), longitud media de frase, número de emblemas, ilustradores y reguladores registrados en la transcripción, número de marcadores discursivos,... Obviamente, esta Base de datos se va completando muy lentamente, debido sobre todo a la complejidad de la transcripción. En el momento de redactar este trabajo, sólo 10 de las 33 transcripciones tienen una versión definitiva, y sólo se han cubierto para todas ellas algunos de los campos. La publicación de los dos primeros volúmenes está prevista para finales de 2005 (Gallardo y Sanmartín, 2005, Gallardo y Moreno, 2005).
} 
- 1975, J. Withney: estrategias comunicativas compensatorias (ECC) para la interacción con hablantes afásicos. Este programa fue propuesto por J. Whitney y desarrollado después por Audrey Holland (1991: 201). A partir de un análisis detenido de la interacción entre el hablante afásico y el logopeda y/o un familiar (cónyuge, por ejemplo), se identifican ciertas estrategias comunicativas que funcionan como mecanismos desencadenantes de mensajes; un segundo paso consiste en la explicitación de estas estrategias (las que son exitosas y las que no) con el hablante afásico y su interlocutor habitual, a fin de poder objetivarlas; en tercer lugar, en los casos en que pueda ser necesario, se realiza una instrucción específica en el uso de tales estrategias compensatorias.

- 1981, Terry Wertz y su equipo realizan una versión del Perfil de Comunicación Funcional de M. T. Sarno (1969), la Escala de Actuación Funcional (Rating of Functional Performance), donde familiares y/o cuidadores informan sobre la eficacia en las habilidades lingüísticas del sujeto.

- 1983, el Protocolo Pragmático de Carol Prutting y Diane Kirchner se realiza a partir de dos conversaciones grabadas, una con un interlocutor de confianza y otra con un interlocutor extraño.

- 1985, PACE: Promoting Aphasic Communicative Effectiveness, de Albyn Davis y Jeanne Wilcox no introduce la triangulación, pero su asunción básica al insistir en la igualdad participativa del logopeda y el cliente.

- 1987. PST, Pragmatics Screening Test, de P. Prinz y F. Weiner, pensado para la evaluación infantil; incorpora también la información de un observador, el maestro del niño, que valora su conducta verbal general.

- 1988. PPECS, Pragmatics Profile of Early Communication Skills, de Hazel Dewart y Susie Summers. Se basa exclusivamente en una entrevista triangulada, donde los padres o cuidadores del niño informan de su conducta.

- 1989. CETI, Communicative Effectiveness Index, de Lomas et al. Se pide a los familiares y allegados del hablante afásico que evalúen su actuación en dieciséis ítems cotidianos, comparándola con la actuación previa a la afasia.

- 1991, EC: Entrenamiento conversacional de Audrey Holland. Se prepara un breve guión monológico (entre 6 y 8 palabras) que debe resultar claramente dificultoso para el hablante en cuestión; el guión se diseña para forzar al paciente a usar estrategias previamente trabajadas. Una vez ha sido preparado, el logopeda y el hablante practican el texto; el logopeda sugiere estrategias para que el hablante logre comunicar el contenido del guión, y lo guía en su ejecución. Después, entra en la sesión un familiar del hablante que no conoce el guión, y el hablante le comunica su contenido con la ayuda del logopeda. También los familiares se entrenan en cómo hacer preguntas, cómo adivinar e interpretar al hablante, etc. Toda la sesión se graba y, cuando el hablante ha terminado de explicar el guión a su familiar, todos ven la grabación y la comentan. Por último, el proceso se repite con la asistencia de una cuarta persona, y nuevamente vuelve a analizarse el vídeo para observar diferencias y establecer la dirección que seguirá el entrenamiento.

- 1997. CAPPA: Conversational Analysis Profile for People with Aphasia de Anne Whitworth, Lisa Perkins y Ruth Lesser. Consta de dos entrevistas al interlocutor- 
clave (aunque puede participar también el hablante afásico según sus posibilidades) y el análisis de una conversación de 10 minutos entre ambos.

- 1995. La Conversación Asistida de Aura Kagan. Sistema desarrollado en el centro de afasia Centre-North de York, en Toronto, desde los años 80 (ahora Centro de Afasia Pat Arato, como homenaje a la mujer que lo inició en 1979, esposa de un afásico). Se trata de un programa de entrenamiento dirigido a personas que se relacionan con hablantes afásicos; en cuatro días se les proporciona herramientas teóricas y estrategias conversacionales dirigidas a mejorar la eficacia comunicativa.

\section{La Guía Comunicativa}

Como hemos dicho, nuestra asistencia y participación en la grabaciones nos llevó a pensar en la necesidad de que los hablantes que mantienen su competencia comunicativa intacta (frente a los hablantes afásicos, cuya competencia aparece enmascarada por la afasia, que los hace parecer in-competentes) podían ser también objeto de atención. La idea fundamental es que se pueden incrementar las habilidades comunicativas del hablante afásico ( $y$ por extensión, su calidad de vida ${ }^{3}$ ) incidiendo en la disponibilidad comunicativa de su entorno inmediato.

Uno de los aspectos donde esta intervención resulta más interesante, y lo trataremos ahora a modo de ejemplo, es el sistema de la toma de turno. Como sabemos, la caracterización etnometodológica del sistema de la toma de turno identificó una serie de rasgos exclusivos ${ }^{4}$ de este acontecimiento comunicativo, y un sistema de reglas que ordenan el cambio de hablante. Este sistema de toma de turno configura la SUPERESTRUCTURA DE LA CONVERSACION, con modificaciones de orden contextual o situacional que nos permiten distinguir tipos de conversación (transaccional e interpersonal, básicamente -Fishman 1971), pero que no alteran esa estructura fundamental. Cuando alguno de los rasgos de la toma de turno conversacional plantea algún tipo de conflicto y, en consecuencia, no se respetan las reglas de cambio de hablante, surge una serie de fenómenos cuya gestión puede plantear problemas al hablante con una competencia alterada; se trata básicamente de:

3 No existen estudios epidemiológicos definitivos sobre la afasia, pero podemos ofrecer datos parciales que nos informan sobre su incidencia, en evidente contraste con su invisibilidad social. Así, a partir de Berthier (2004) podemos extraer los siguientes datos referidos específicamente a afasias causadas por ACV y a su incidencia anual en Europa, en las edades entre 25-74 años (no se incluyen los TCE, tumores y otras causas): hombres: 318 372/100.000 habitantes, mujeres: $195-240 / 100.000$ habitantes. Esto supone una incidencia entre el 21 y el $38 \%$ sólo de afasias por ACV.

Respecto a los TCE, se calcula (http://salud.discapnet.es) una incidencia general de 200 casos/100.000 habitantes, con las siguientes matizaciones: Varones: tres veces más frecuente que en mujeres. Edad: mayor frecuencia entre los 15 y los 29 años. Atropellos y caídas, más frecuentes en niños y mayores de 65 años. Un tercio aproximadamente desarrollan afasia.

4 Estos son los 14 rasgos definitorios de esta toma de turno conversacional (Sacks, Schegloff, Jefferson 1974, 699): 1) cambio(s) de hablantes recurrente(s); 2) en general, cada vez habla un solo participante; 3) los solapamientos (habla simultánea) son frecuentes pero breves; 4) con frecuencia, las transiciones entre los turnos no son espaciadas; 5) el orden de los turnos no es fijo, sino variable; 6) la duración de los turnos no es fija, sino variable; 7) la longitud de la conversación no se especifica previamente; 8) lo que dicen las partes no se especifica previamente; 9) la distribución relativa de los turnos no se especifica previamente; 10) el número de participantes puede variar; 11) el habla puede ser continua o discontinua; 12) existen técnicas de distribución del turno; 13) se utilizan distintas unidades construccionales del turno; y 14) hay mecanismos de rectificación para los errores y violaciones de la toma de turno. 
- el solapamiento

- las escisiones y cruces conversacionales

- el turno colaborativo y el turno competitivo.

Como veremos, en estas situaciones conversacionales, la conducta del interlocutor clave resulta esencial, pues su actitud (cuando interrumpe, cuando solapa, cuando contesta en lugar del hablante afásico, o cuando lo acosa con preguntas) está casi siempre al filo de la doble interpretación. Efectivamente, si en el ámbito de la interacción cotidiana nos hacen una pregunta y en nuestro lugar interviene otra persona, esa intervención que interfiere está diciendo algo sobre nuestra capacidad de responder, pero en el caso de la conversación afásica cabe señalar que esa interferencia, simultáneamente, está impidiendo que nuestra actuación pueda hacer demasiado evidente que no somos capaces de dar la respuesta... Aquí es necesario tener en cuenta que se crea una especie de "identidad compartida" o "identidad cómplice" entre el sujeto afásico y el interlocutor clave... ¿Y cómo distinguir la línea ténue que separa estas dos interpretaciones y, por extensión, la identidad individual del sujeto afásico y la identidad cómplice que el interlocutor clave pretende compartir con él al hablar en su lugar? Esta posición obedece a la especial situación de dependencia que ha creado la afasia, y que casi siempre es novedosa para ambos participantes.

El solapamiento es la superposición de dos o más intervenciones. El sistema de toma de turno conversacional establece que existen solapamientos frecuentes, pero que son breves:

- la frecuencia puede tener distinto valor según las culturas; se dice, por ejemplo, que las culturas latinas son más dadas a la interrupción y el solapamiento que las culturas anglosajonas, algo en lo que influye no sólo un diferente criterio de cortesía sino también la estructura sintáctica de las lenguas en cuestión.

- la brevedad está condicionada por nuestra limitada capacidad para escuchar (y entender) dos cosas a la vez.

Cuando estudiamos el fenómeno del solapamiento en la conversación cotidiana, observamos algunas conductas repetitivas por parte de los hablantes; por ejemplo, vemos que se produce un solapamiento sistemático de ciertas estructuras finales de la intervención, que el hablante 1 utiliza para ceder el turno al hablante 2 (por ejemplo preguntas añadidas (tag questions) como “¿eh?”, “¿no?”):

074 T. vull que lo repetixca/ $[i$ eh?

$075 \mathrm{P}$.

[sí/ ja ho sé (ASENTIMIENTO CON LA CABEZA)

[Corpus PerLA, 02, SBW. 074-075]

En otras ocasiones el solapamiento ocasiona estructuras repetidas al inicio de las intervenciones; los estudios han demostrado que cuando un hablante "roba el turno", suele repetir la parte inicial de su intervención (REINICIOs), hasta que esté seguro de que se le escucha. Las grabaciones de vídeo demuestran, además, que el hablante deja de repetir cuando consigue atraer la mirada de los participantes y, por tanto, verifica su escucha. En el siguiente fragmento, la hablante afásica $(\mathrm{P})$ conversa con su hijo $(\mathrm{H})$ :

013 H.pero no le digas esoo/ porque cuando tú salistes del Clínico/ cuando te dieron el alta $\uparrow /$ en enero $\uparrow$

014 P. ya

015 H.tú no hablabas casi nada $\uparrow / /$ y ahora charras por los codos/ tú hablas mucho^ 


\section{P. [claro- claro ((que hablo)) \\ 017 H. [lo que pasa es ahoraa/] te ves un pocooo \\ [Corpus PerLA, JFG, 013-017]}

Como se puede apreciar, estos dos fenómenos (el solapamiento de la frase de cesión de turno, y el reinicio en el turno de autoselección) son consecuencia directa de las reglas que, según hemos descrito, gobiernan el cambio de hablante.

En la conversación con hablantes afásicos hay que ser cuidadoso para que los solapamientos se limiten a estas dos situaciones automáticas; es necesario ser muy conscientes de otro tipo de situaciones en que más de dos hablantes tratan de intervenir simultáneamente, y que se prolongan durante un espacio de tiempo que supera la capacidad de entendimiento de cualquier hablante. Frente a los casos anteriores, existen dos tipos de solapamiento que no son propiciados por la gestión del turno, sino que constituyen una verdadera interrupción (Gallardo 1993):

- El turno colaborativo: es un turno que se construye en solapamiento y que intenta completar el turno del interlocutor; en la conversación cotidiana, sus motivaciones suelen de ser refuerzo de la imagen de uno o los dos participantes: o bien ayudamos al hablante a completar un turno que le resultaba problemático (a veces por falta de información), o bien lo terminamos por él para demostrar empatía, pues somos capaces de adelantarnos a sus palabras (es una versión máxima de la ratificación o la conformidad).

- El turno competitivo: es una interrupción que intenta arrebatar el turno al hablante que posee la palabra, y suele construirse por eso con un tono de voz más fuerte de lo normal; es frecuente, además, que se construyan reinicios hasta que efectivamente se posee la palabra y se asegura la recepción.

En la conversación afásica, el interlocutor clave construye turnos competitivos y colaborativos que, en la mayoría de los casos, intentan facilitar las cosas al hablante afásico; lo que ocurre es que en ocasiones ese facilitamiento puede ser interpretado de diversas maneras. No es fácil encontrar el equilibrio entre lo que resulta necesario para facilitar la comprensión y lo que resulta excesivo (amenazante u ofensivo) para el afásico. Lo que, por ejemplo, en una conversación habitual se interpretaría como turno colaborativo, en el marco de la conversación afásica puede ser interpretado como un acto de MINORACIÓN. Con este término, procedente de la pragmática intercultural, nos referimos a las conductas del hablante no afásico que pueden ser interpretadas como reveladoras de una actitud peyorativa o, a veces, excesivamente proteccionista. Fruto de esta misma intención colaborativa aparecen otros fenómenos de doble interpretación, como la sucesión abrumadora de preguntas, que en lugar de ayudar o dar pistas al afásico, lo confunden y distraen. Lo que los etnometodólogos llaman "versiones de insistencia", es decir, intervenciones que tratan de obtener una reacción prioritaria por parte del interlocutor, se suceden rápidamente sin dar hueco posible para que el hablante afásico tome la palabra.

Junto a las interrupciones conversacionales, en las situaciones en que hay más de dos conversadores, pueden producirse otros dos tipos de fenómenos que entendemos que pueden ser amenazantes para la identidad del sujeto afásico:

- la escisión conversacional se produce cuando se generan dos conversaciones simultáneas (subdiálogos); nuestros datos ofrecen ejemplos de escisiones cuando a veces el 
entrevistador conversa aisladamente con el interlocutor-clave, colocando al hablante afásico en una situación de casi "invisibilidad".

- el cruce conversacional se da cuando, dada una situación previa de escisión, un hablante que mantiene un subdiálogo con otro, interviene ocasionalmente en otro intercambio. No es una situación frecuente en nuestro corpus, y tampoco resulta especialmente amenazante.

Una versión especialmente frecuente de la escisión conversacional se da cuando los hablantes no afásicos conversan sobre el afásico en su presencia, pero sin considerarlo como participante. Otra situación que se repite es la de interferencias en la respuesta: el entrevistador hace preguntas dirigidas al hablante afásico, pero mientras este responde 0 antes de que lo haga, interviene el interlocutor clave. Hay que entender que en la mayoría de los casos, la pretensión sigue siendo igualmente colaborativa; la hija/madre/esposa/ nieto... intentan facilitar las cosas al afásico, y si responden por él se evitan y le evitan la situación angustiosa que pone de manifiesto el déficit. Es necesario un ejercicio breve de reflexión metalingüística para que el interlocutor-clave se dé cuenta de esta situación y las consecuencias que puede tener para el futuro.

\section{Conclusiones}

Todas estas reflexiones son, como venimos diciendo, las que nos han llevado a diseñar una Guía Comunicativa para la Conversación Afásica (Gallardo 2005b). El logro fundamental que persigue tal Guía es la OBJETIVACIÓN de las conductas conversacionales, a fin de poder luego transformarlas; después de un recorrido breve por las categorías pragmáticas básicas, la Guía ofrece una serie de técnicas específicas que pueden resultar útiles al hablante que se relaciona con interlocutores afásicos, por ejemplo el uso de los silencios, el establecimiento de señales-testigo, el uso del desembrague conversacional... El proceso parte del análisis del corpus: observamos las grabaciones, identificamos las conductas del interlocutor-clave, y analizamos sus consecuencias positivas o negativas en la interacción. En la Guía se presentan las conductas que pueden ser facilitadoras y se insiste en las que pueden ser entorpecedoras y/o minorizantes. Su aplicación se completa, además, con el diseño de Cursos de habilidades comunicativas para interlocutores-clave, que tratan de proporcionar un marco efectivo para la presentación y práctica de estas técnicas ${ }^{5}$.

\section{Referencias bibliográficas}

Barthes, Roland (1973): "La división de los lenguajes". En El susurro del lenguaje. Más allá de la palabra y la escritura. Barcelona, Paidós, 1987. Trad. de C. Fernández Medrano.

Baudrillard, Jean (1987): El otro por si mismo. Barcelona, Anagrama, 1988. Trad. de Joaquín Jordá.

Benveniste, Émile (1958): "De la subjetividad en el lenguaje", en (1966): Problemas de Lingüistica General, vol 1. México, Siglo XXI, 1991, págs. 179-189. Trad. española de Juan Almela.

5 El primero de estos Cursos, coorganizado por el Vicerrectorado de Relaciones con la Sociedad y el departamento de Teoría de los Lenguajes de la Universitat de València, con el Servicio de Daño Cerebral de Hospitales NISA, se celebró en septiembre de 2005 en el Hospital Valencia al Mar, impartido por Beatriz Gallardo Paúls y Raúl Villodre Campos. 
Benveniste, Émile (1970): "Estructura de la lengua y estructura de la sociedad", en (1966): Problemas de Lingǘstica General, vol. 2. México, Siglo XXI, 1991, págs. 95-106. Trad. española de Juan Almela.

Berthier, Marcelo L. (2004): "Nuevas estrategias en el tratamiento de la afasia crónica postictus: análisis preliminar de eficacia y seguridad del donepezilo", Inv. Clin. Farm. 2004, Vol. 1 (3), págs. 09-17.

Brown, Roger; Gilman, Albert (1960): "The pronouns of power and solidarity". En Fishman, J., ed: Readings in the Sociology of Languag. The Hague, Mouton, 1968, págs. 252-275.

Caporali, Alessandra; Basso, Anna (2003): "A survey of long-term outcome of aphasia and of chance of gainful employment", Aphasiology 17 (9), págs. 815-834.

Dewart, Hazel; Summers, Susie (1995): Pragmatics Profile of Everyday Communication Skills in Children. Windsor, Nfer-Nelson.

Fairclough, Norman (1992): Discourse and social change. Cambridge, Polity Press.

Gallardo Paúls, Beatriz (2004): "La construcción de la identidad patológica en la interacción cotidiana". En El joc de les identitats en discursos orals, Jornada de tardor 2004 organitzada per la Xarxa d'Estudis del Discurs i la Xarxa sobre l'Educació Lingüística i la Formació d'Ensenyaments en Contextos Multilingües i Multiculturals, Barcelona 1 i 2 d'octubre de 2004.

Gallardo Paúls, Beatriz (2005a): "El lenguaje y la mente". En A. López García y B. Gallardo Paúls (Eds.): Conocimiento y lenguaje. Valencia, Universitat de València.

Gallardo Paúls, Beatriz (2005b): Afasia y conversación: las habilidades comunicativas del interlocutor clave. Valencia, Tirant Lo Blanch, 2005.

Gallardo Paúls, Beatriz; Sanmartín Sáez, Julia (2005): Afasia fluente. Materiales para su estudio. Valencia, Universitat/AVaLCC.

Gallardo Paúls, Beatriz; Moreno Campos, Verónica (2005): Afasia no fuente. Materiales y análisis pragmático. Valencia, Universitat/AVaLCC..

Galván, Fernando (1997): "Intertextualidad o subversión domesticada: aportaciones de Kristeva, Mai y Plett”. En M. Bengoechea y R. Sola (Eds): Intertextuality/ Intertextualidad. Alcalá, Universidad, págs. 35-80.

Garrett, Kathryn L. (2003): "Strategy use in context': AAC, Supported conversation, and Group Therapy Interventions for People with Severe Aphasia". En http:/aac.unl.edu/drb/garret/garoutv. pdf (septiembre 2003).

Giddens, Anthony (1991): Modernidad e identidad del yo. El yo y la sociedad en la época contemporánea. Barcelona, Península, 1997. Trad. de José Luis Gil.

Goffman, Erving (1959): La presentación del yo en la vida cotidiana. Buenos Aires, Amorrortu, 1987. Trad. de Hildegarde B. Torres y Flora Setaro.

Golopentjia, Sanda (1988): "Interaction et histoire conversationnelles". En Cosnier, Gelas, Kerbrat, eds: Echanges sur la conversation. Paris, CNRS, págs. 69-82.

Gumperz, John J. (1968): "Types of Linguistic Communities". En J. Fishman, Ed: Readings in the Sociology of language. The Hague, Mouton, 1972, págs. 460-476.

Habermas, Jürgen (1981): Teoría de la acción Comunicativa. Vol 1 : Racionalidad de la acción y racionalización social. Madrid, Taurus, 1987. Trad. de Manuel Jiménez Redondo.

Jorques, Daniel (1997): Interpelación y espacios comunicativos. Valencia, Universitat.

Kagan, Aura (1995): "Revealing the competence of aphasic adults through conversation: a challenge to health professionals", Topics in Stroke Rehabilitation, 2, págs. 15-28.

Kagan, Aura (1998): "Supported Conversation for Adults with Aphasia: Methods and Resources for Training Conversation Partners", Aphasiology, 12, 9, Sept., págs. 816-830.

Kauhanen, Marja-Liisa (1999): Quality of life after stroke. Clinical, functional, psychosocial and cognitive correlates. University of Oulu, Oulu (Finlandia).

Kerbrat-Orecchioni, Catherine (1987): La enunciación. De la subjetividad en el lenguaje. Buenos Aires, Hachette, 1986. Traducción de Gladys Anfora y Emma Gregores. 
Kristeva, J. (1986a): "Word, dialogue and novel". En T. Moi (ed): The Kristeva Reader. Oxford, Basil Blackwell, págs. 24-33.

Kristeva, J. (1986b): "The system of the speaking subject". En T. Moi (ed): The Kristeva Reader. Oxford, Basil Blackwell, págs. 34-61.

Parr, Susie; Byng, Sally; Gilpin, Sue (1997): Talking about aphasia. Mainenhead, Philadelphia, Open Univ. Press.

Prinz; Wiener (1987): The Pragmatics Screening Test, San Antonio, TX, The Psychological Corporation.

Rodríguez Bausá, Luis (2003): "La imagen del otro en relación a la discapacidad. Reflexiones sobre la alteridad", http://www.uclm.es/profesorado/ricardo/Docencia_e_Investigacion/2003.htm (oct.2004), Docencia e Investigación, num 3.

Saville-Troike, Muriel (1989): The Ethnography of Communication. Cambridge, MA, Blackwell.

Sennett, Richard (1976): El declive del hombre píblico. Barcelona, Península, 1978. Trad. de Gerardo Di Masso.

Tinti, Alessandra; Marogna, Maura (2000): "Riabilitare l'afasia: una questione di parole?", www. aphasiaforum.com/Gengll.htm (julio 2004).

Whitworth, Anne; Perkins, Lisa; Lesser, Ruth (1997): Conversational Analysis Profile for People with Aphasia. Whurr Publ., London. 